\title{
An overview of neural networks use in anomaly intrusion detection systems
}

\begin{abstract}
With the increasing number of computers being connected to the Internet, security of an information system has never been more urgent. Because no system can be absolutely secure, the timely and accurate detection of intrusions is necessary. This is the reason of an entire area of research, called Intrusion Detection Systems (IDS). Anomaly systems detect intrusions by searching for an abnormal system activity. But the main problem of anomaly detection IDS is that; it is very difficult to build, because of the difficulty in defining what is normal and what is abnormal. Neural network with its ability of learning has become one of the most promising techniques to solve this problem. This paper presents an overview of neural networks and their use in building anomaly intrusion systems.
\end{abstract}

Keyword: Anomaly detection; Intrusion detection systems; Neural network 